\title{
Weed Biomass and Weed Species Diversity of Juvenile Citrus Trees Intercrop with some Arable Crops
}

\author{
Patience Mojibade OLORUNMAIYE*, Stephen Olaoye AFOLAYAN² \\ ${ }^{1}$ University of Agriculture Abeokuta, Department of Plant Physiology and Crop Production, \\ Abeokuta,Nigeria;pmmoji@yahoo.co.uk ("corresponding author) \\ ${ }^{2}$ National Horticultural Research Institute, Bagauda Substation, Kano, Nigeria
}

\begin{abstract}
A preliminary study was carried out to evaluate the performances of eight crops in the intercrop of citrus with arable crops at the National Horticultural Research Institute (NIHORT) Ibadan, Nigeria. Eight arable crops: maize, cucumber, sweet potato, Corchorus olitorius, large green, grain amaranth, Mucuna pruriens var. utilis, and groundnut were intercropped with young citrus trees in the early planting season of 2010 with sole citrus as control. The experiment was laid out in a completely randomized block design with three replicates. Data were collected on weed flora, weed density and weed dry weight. Results showed that the relative frequencies of weeds in all the plots were less than 4\% at both 6 and 9 WAP. Gomphrena celosoides, Oldenlandia corymbosa and Tridax procumbens were most preponderant in appearing in all the plots. Tridax procumbens had a consistent relative frequency $(2.34 \%)$ in all the plots except in citrus/ maize plot $(0.78 \%)$ at 9 WAP. Significantly lower broadleaf weed densities were obtained in citrus/sweet potato, citrus/large green, control plot and citrus/cucumber $\left(28.67,45.00,50.00\right.$ and $76.33 \mathrm{~m}^{-2}$ respectively) than in citrus/groundnut plot $\left(143.00 \mathrm{~m}^{-2}\right)$. Similarly, significantly lower grass weed densities were produced in citrus/Mucuna and citrus/sweet potato $\left(0.33 \mathrm{~m}^{-2}\right.$ each $)$ plots than the control plot $\left(11.33 \mathrm{~m}^{-2}\right)$. Whereas citrus/Corchorus plot produced significantly lower broadleaf weed dry weight $\left(37.59 \mathrm{~g} \mathrm{~m}^{-2}\right)$ than citrus/ Mucuna plot $\left(126.47 \mathrm{~g} \mathrm{~m}^{-2}\right)$ at 3 WAP, citrus/large green plot $\left(16.15 \mathrm{~g} \mathrm{~m}^{-2}\right)$ and citrus/groundnut plot $\left(123.25 \mathrm{~g} \mathrm{~m}^{-2}\right)$ followed the same trend at 6 WAP. Sedges dry weights were less than $7 \mathrm{~g} \mathrm{~m}^{-2}$ in all the plots compared with control plot.
\end{abstract}

Keywords: weed density, relative frequency, Mucuna pruriens, large green, grain amaranth, cucumber

\section{Introduction}

Citrus as a fruit crop acts as a rich source of vitamin $\mathrm{C}$ and other essential nutrients required by human body and this has necessitated its wide cultivation in Nigeria (Adewale et al., 1996), Brazil, USA and other countries (Florida Citrus Agriculture, 2004). The citrus trees take longer time to produce fruit and so short duration crops like chilli, amaranth (Aiyelaagbe, 2001), turmeric, pepper, ginger and basil (Oyedele et al., 2011) can provide ready cash flow. In the southern part of Nigeria, a number of crops like cowpea, okra, water melon, pepper, amaranth and maize have been found to be compatible with citrus intercrop (NIHORT, 2000). Intercropping is the cultivation of two or more crops at the same time in the same field. Its profits are risk minimization, increase in farmers' income and food security, reduction of soil erosion, pest and disease control (Bekunda, 1999; Bekunda and Woomer, 1996; Jodha, 1979; Owuor et al., 2002). Depending on the crops intercropped, competition for water, light and nutrients may result in lower yields. Lower weed biomass has been reported in intercropping systems where a main crop was inter-sown with a "smother" crop species (Jodha, 1979). Greater crop yield and less weed growth can be obtained more frequently in intercrops than in sole crops. In
East Africa fruit crops are usually intercropped with annual crops for example, banana is intercropped with food and / or fodder crops (Clark and Francis, 1985) while in India bananas are intercropped with potato which had resulted in good returns (Okigbo, 1979). An intercrop between citrus mandarin seedlings and cucumber has also been reported to give high yield of cucumber fruit and minimal interference in the growth of citrus seedlings which might be due to the low growing nature of the latter (Natarajaa and Nairk, 1992). In Kenya, fruit trees are intercropped with all types of short term crops such as beans, peas, potatoes, maize, millet, exotic and indigenous vegetables when they are still young as a way of attaining food security and income before the trees mature. Intercropping can be used as an effective weed control strategy (George and Jeruto, 2010) as different plant types growing together enhances weed control by increasing shade and crop competition with weeds through tighter crop spacing. Data on the weed biomass and weed flora diversity in juvenile citrus intercrop with arable crops at the National Horticultural Research Institute, Ibadan, a south-west forest ecological zone of Nigeria has not been documented. It is therefore the objective of this study to investigate the weed biomass, weed density and weed species diversity of a citrus intercrop with some arable crops. 
132

\section{Materials and methods}

The study was conducted at National Horticultural Research Institute (NIHORT), Ibadan, Nigeria in the early cropping season of 2010. The treatments comprised of 8 arable crops [maize (var. 'DMR LSR' yellow)], cucumber (royal hybrid F1), sweet potato, Corchorus olitorius, large green, grain amaranth, Mucuna pruriens var. utilis and groundnut) with a citrus plot without any crop as control.
The arable crops were intercropped with the young citrus trees of about 3 years old. Land preparation was done manually by slashing with cutlass in late April, 2010 and the experiment was laid in a completely randomized block design with three replicates. The size of each plot was $6 \mathrm{~m}$ $\times 12 \mathrm{~m}$. Maize and M. pruriens were planted at a spacing of $90 \mathrm{~cm} \times 45 \mathrm{~cm}$ each, cucumber, groundnut and sweet potato at $60 \mathrm{~cm} \times 30 \mathrm{~cm}$ each while Corchorus, large green, and grain amaranth were drilled at $30 \mathrm{~cm}$ apart each at a

Tab. 1. Weed species composition of intercropping citrus with arable crops in NIHORT, Ibadan Nigeria

\begin{tabular}{|c|c|c|}
\hline Weed species & Growth form & Families \\
\hline Acroceras zizanoides Dandy & PG & Poaceae \\
\hline Ageratum conyzoides L. & $\mathrm{ABL}$ & Asteraceae \\
\hline Alternanthera sessilis (L.) R. Br. Ex Roth & $\mathrm{ABL}$ & Amaranthaceae \\
\hline Amaranthus viridus & $\mathrm{ABL}$ & Amaranthaceae \\
\hline Aspilia africana (Pers.)c. Adams & $\mathrm{ABL}$ & Asteraceae \\
\hline Axonopus compressus (Sw.) P. Beauv. & AG & Poaceae \\
\hline Boerhavia diffusa $\mathrm{L}$. & $\mathrm{ABL}$ & Nyctaginaceae \\
\hline Celosia spp. & $\mathrm{ABL}$ & Amaranthaceae \\
\hline Centrosema pubescens Benth. & PBL & Fabaceae \\
\hline Cleome viscossa & $\mathrm{ABL}$ & Cleomaceae \\
\hline Commelina benghalensis $\mathrm{L}$. & $\mathrm{ABL}$ & Commelinaceae \\
\hline Commelina erecta L. & $\mathrm{ABL}$ & Commelinaceae \\
\hline Corchorus olitorious L & $\mathrm{ABL}$ & Tiliaceae \\
\hline Cyperus esculentus Linn. & PS & Cyperaceae \\
\hline Cynodon dactylon & PG & Poaceae \\
\hline Desmodium scropiurus (Sw.) Desv. & $\mathrm{ABL}$ & Fabaceae \\
\hline Digitaria horizontalis & AG & Poaceae \\
\hline Euphorbia heterophylla L. & $\mathrm{ABL}$ & Euphorbiaceae \\
\hline Euphorbia hirta L. & $\mathrm{ABL}$ & Euphorbiaceae \\
\hline Gomphrena celosoides Mart. & $\mathrm{ABL}$ & Amaranthaceae \\
\hline Ipomoea bartatas & $\mathrm{ABL}$ & Convolvulaceae \\
\hline Ipomoea triloba Linn. & $\mathrm{ABL}$ & Convolvulaceae \\
\hline Larpotea aestuans (Linn.) Chew. & $\mathrm{ABL}$ & Urticaceae \\
\hline Leptochloa caerulescens & AG & Poaceae \\
\hline Mariscus alternifolius Vahl & PS & Cyperaceae \\
\hline Mitracarpus villosus (SW) DC & $\mathrm{ABL}$ & Rubiaceae \\
\hline Oldenladia corymbosa (Linn.)Roxb. & $\mathrm{ABL}$ & Rubiaceae \\
\hline Panicum maximum Jacq. O. Ktze & AG & Poaceae \\
\hline Paspalum obiculare Forst. & AG & Poaceae \\
\hline Paspalum conjugatum Berg & AG & Poaceae \\
\hline Peperomia pellucida Linn & $\mathrm{ABL}$ & Piperaceae \\
\hline Phillanthus amarus Schum, et Thonn. & $\mathrm{ABL}$ & Euphorbiaceae \\
\hline Platostoma africanum P. Beauv. & $\mathrm{ABL}$ & Lamiaceae \\
\hline Portulaca oleracea Linn. & $\mathrm{ABL}$ & Portulaceae \\
\hline Physalis angulata Linn. & $\mathrm{ABL}$ & Solanaceae \\
\hline Setaria barbata (Lam.) Kunth. & AG & Poaceae \\
\hline Setaria longiseta & AG & Poaceae \\
\hline Senna spp. & $\mathrm{ABL}$ & Caesalpiniaceae \\
\hline Synedrella nodiflora Gaertn. & $\mathrm{ABL}$ & Asteraceae \\
\hline Talinum triangulare (Jacq.) Willd. & PBL & Portulaceae \\
\hline Tridax procumbens $\mathrm{L}$. & $\mathrm{ABL}$ & Asteraceae \\
\hline Waltheria indica $\mathrm{L}$. & PBL & Sterculiaceae \\
\hline
\end{tabular}

Note: AG = Annual Grass; PBL = Perennial Broadleaf; PG = Perennial Grass; PS = Perennial Sedge; ABL = Annual Broadleaf 
seed rate of $6 \mathrm{~kg} \mathrm{ha}^{-1}$ between 29 April and 13 May. All the crops were planted at $1 \mathrm{~m}$ away from the base of the young citrus trees. Hoe weeding was done 2 times. Data were collected on weed density, weed dry weight and weed flora at 3,6 and 9 WAP with the use of two $0.5 \mathrm{~m} \times 0.5 \mathrm{~m}$ quadrate which were permanently placed randomly within each plot. Weeds within each quadrate were uprooted, sorted into broadleaves, grasses and sedges, identified, counted, and recorded to compute weed density. The weeds were then oven dried for 48 hours at $80^{\circ} \mathrm{C}$ and weighed to compute weed dry weight. Data on weed density and weed dry weight were subjected to analysis of variance using Genstat package while ecological analysis of the weed flora was carried out to determine the weeds relative frequencies.

\section{Results}

\section{Relative frequencies of weeds}

A total of 42 weed species belonging to 19 families were encountered in the course of this study (Tab. 1). Poaceae family had 10 species, Amaranthaceae and Asteraceae had 4 each, Euphorbiaceae 3, Commelinaceae, Convolvulaceae, Cyperaceae, Fabaceae, Portulaceae and Rubiaceae had 2 each while Caesalpinaceae, Cleomaceae, Lamiaceae, Piperaceae, Tiliaceae, Solanaceae, Sterculiaceae Urticaceae and Nyctaginaceae had 1 species each. There were 30 broadleaf weed species, 10 grasses and 2 sedges. At 6 WAP, citrus/ Corchorus plot had the highest number of weed species (19) followed by groundnut plot (17), citrus plots inter-

Tab. 2. Weed relative frequencies (\%) in young citrus trees intercropped with arable crops 6 WAP at NIHORT, Ibadan, Nigeria

\begin{tabular}{|c|c|c|c|c|c|c|c|c|c|}
\hline \multirow{2}{*}{ Weed species } & \multicolumn{9}{|c|}{ Relative frequency (\%) 6 WAP } \\
\hline & MA & $\mathrm{CU}$ & $\mathrm{SP}$ & $\mathrm{CO}$ & LG & GA & $\mathrm{MU}$ & GN & $\mathrm{C}$ \\
\hline Acroceras zizanoides Dandy & - & - & - & 0.46 & - & - & - & - & - \\
\hline Ageratum conyzoides L. & 0.46 & 0.92 & 0.46 & 0.92 & 0.46 & 0.46 & - & 0.46 & 0.46 \\
\hline Alternanthera sessilis & 0.92 & 0.46 & 0.92 & 0.46 & 0.46 & - & - & 0.92 & 0.46 \\
\hline Amaranthus viridus & - & - & 0.92 & - & 0.46 & 1.38 & 0.46 & 0.46 & - \\
\hline Aspilia africana (Pers.) c. Adams & - & - & - & - & - & 0.46 & - & - & - \\
\hline Axonopus compressus (Sw.) P. Beauv. & - & 0.46 & - & - & 0.46 & - & - & - & - \\
\hline Boerhavia diffusa L. & - & - & - & 0.46 & - & 0.46 & - & - & - \\
\hline Centrosema pubescens Benth. & - & - & & & & & & & \\
\hline Commelina benghalensis L. & 0.92 & 0.46 & 0.46 & - & - & 0.46 & - & 0.46 & 1.38 \\
\hline Corchorus olitorious Linn. & 0.92 & 0.46 & - & 0.92 & 0.92 & - & 0.92 & 0.46 & - \\
\hline Cyperus esculentus Linn. & 0.46 & 0.92 & - & - & - & - & - & 0.46 & 0.46 \\
\hline Desmodium scropiurus (Sw.) Desv. & 0.46 & - & - & - & - & - & 0.46 & 0.46 & 0.46 \\
\hline Euphorbia heterophylla Linn. & - & 0.46 & 0.46 & - & - & - & 0.46 & 0.46 & - \\
\hline Euphorbia hirta Linn. & 0.46 & 0.46 & - & 0.46 & - & 0.46 & 0.46 & - & - \\
\hline Gomphrena celosoides Mart. & 0.92 & 1.38 & & & & & & & \\
\hline Ipomoea triloba Linn. & - & 0.46 & 0.46 & - & - & - & - & - & - \\
\hline Larpotea aestuans (Linn.) Chew. & - & - & - & 0.92 & - & 0.92 & - & - & - \\
\hline Leptochloa caerulescens Steud & - & - & - & 0.92 & 1.38 & 1.38 & 0.46 & 0.92 & 0.92 \\
\hline Mariscus alternifolius Vahl & -0.46 & 1.38 & - & 0.92 & 0.46 & 0.92 & 1.38 & 1.38 & 1.38 \\
\hline Mitracarpus villosus (SW) DC & - & - & 0.46 & 0.46 & $0.92-$ & - & - & - & - \\
\hline Oldenladia corymbosa (Linn.)Roxb. & - & - & - & 0.46 & 0.92 & 0.92 & - & - & - \\
\hline Panicum maximum Jacq. O. Ktze & - & - & - & - & - & - & - & 0.46 & - \\
\hline Peperomia pellucida (L.) H. B. \& K. & - & - & - & - & 0.92 & - & - & - & - \\
\hline Phillanthus amarus Schum, et Thonn. & - & - & - & 0.46 & 0.46 & 0.46 & - & - & - \\
\hline Portulaca oleracea Linn. & - & - & - & 0.46 & - & - & - & - & - \\
\hline Physalis angulata Linn. & - & - & - & 0.46 & - & - & - & - & - \\
\hline Senna spp. & - & - & - & - & - & - & - & 0.46 & - \\
\hline Setaria barbata (Lam.) Kunth. & - & 0.46 & - & 0.92 & 0.46 & - & - & 1.38 & 0.92 \\
\hline Sida cordifolia Linn. & - & - & - & - & - & - & - & - & 0.46 \\
\hline Spigelia anthelmia Linn. & - & 0.46 & 0.46 & 0.46 & 0.46 & 0.38 & 0.46 & 0.92 & - \\
\hline Synedrella nodiflora Gaertn. & - & - & 0.92 & 0.46 & - & 0.46 & - & 0.46 & - \\
\hline Talinum triangulare (Jacq.) Willd. & 1.38 & 1.38 & 1.38 & 0.92 & 0.92 & 0.46 & 0.46 & 1.38 & 1.38 \\
\hline Tridax procumbens L. & 1.38 & 1.38 & 1.38 & 0.92 & 0.92 & 1.38 & 1.38 & 0.92 & 0.92 \\
\hline Total number of weed species & 11 & 15 & 11 & 19 & 15 & 15 & 10 & 17 & 11 \\
\hline
\end{tabular}

Note: MA = Maize; CU = Cucumber; $\mathrm{SP}=$ Sweet Potato; CO = Corchorus; LG = Large Green; GA = Grain Amaranth; MU = Mucuna pruriens var. utilis; $\mathrm{GN}=$ Groundnut $\mathrm{C}=$ Control 
134

cropped with cucumber, large green and grain amaranth had 15 weed species each, citrus/maize, citrus/sweet potato and control plots had 11 weed species each while citrus/Mucuna plot had 10 (Tab. 2). Two weed species: Talinum triangulare and Tridax procumbens associated with all the crops. Whereas T. triangulare had a consistent relative frequency of $1.38 \%$ in citrus/maize, citrus/cucumber, citrus/sweet potato, citrus/groundnut and control plots, T. procumbens had the same trend in citrus/maize, citrus/ cucumber, citrus/sweet potato, citrus/large green and citrus/grain amaranth plots at 6 WAP (Tab. 2). At 9 WAP, citrus plots intercropped with large green and cucumber recorded 16 weed species each, citrus/maize had 15 weed species, citrus/sweet potato and citrus/Mucuna plots had 14 weed species each, citrus/large green and citrus/ groundnut plots recorded 12 weed species each while the control plot followed the same trend as in 6 WAP (Tab. 3 ). The relative frequencies of weeds in all the plots were less than $4 \%$ at both 6 and 9 WAP (Tab. 2 and 3 ) and at 9 WAP, three weed species: $G$. celosoides, $O$. corymbosa and T. procumbens appeared in all the plots. Tridax procumbens had a consistent relative frequency of $2.34 \%$ in all the plots except in maize plot $(0.78 \%)$ as shown in Tab. 3 .

Tab. 3. Weed Relative Frequencies (\%) in young citrus trees intercropped with arable crops 9 WAP at NIHORT, Ibadan, Nigeria

\begin{tabular}{|c|c|c|c|c|c|c|c|c|c|}
\hline \multirow{2}{*}{ Weed species } & \multicolumn{9}{|c|}{ Relative Frequency (\%) 9 WAP } \\
\hline & MA & $\mathrm{CU}$ & SP & $\mathrm{CO}$ & LG & GA & MU & GN & $\mathrm{C}$ \\
\hline Acroceras zizanoides Dandy & - & 0.78 & - & - & - & - & - & - & - \\
\hline Ageratum conyzoides $\mathrm{L}$. & 0.78 & 0.78 & - & 2.34 & 2.34 & 0.78 & 2.34 & - & 0.78 \\
\hline Alternanthera sessilis (L.) R. ex Roth & - & 1.56 & 1.56 & - & 1.56 & - & 1.56 & 0.78 & 1.56 \\
\hline Amaranthus viridus & 0.78 & 0.78 & 0.78 & - & - & - & - & - & - \\
\hline Aspilia africana (Pers.) c. Adams & - & 0.78 & - & - & - & - & - & - & - \\
\hline Boerhavia diffusa $\mathrm{L}$. & - & - & 0.78 & - & 0.78 & - & 0.78 & 0.78 & - \\
\hline Celosia laxa Schum and Thonn. & - & - & 0.78 & - & - & - & - & - & - \\
\hline Centrosema pubescens Benth. & - & - & - & - & - & 0.78 & - & - & - \\
\hline Cleome viscossa $\mathrm{L}$. & - & - & - & - & 0.78 & 0.78 & - & - & - \\
\hline Commelina benghalensis $\mathrm{L}$. & - & - & - & 0.78 & - & - & - & - & - \\
\hline Commelina erecta L. & - & - & - & - & - & - & - & 0.78 & - \\
\hline Corchorus olitorious L. & 0.78 & 0.78 & - & 0.78 & 1.56 & 0.78 & 0.78 & - & - \\
\hline Cyperus esculentus Linn. & 0.78 & - & 0.78 & - & - & 1.56 & - & 0.78 & 1.56 \\
\hline Cynodon dactylon (Linn.) Pers. & - & 0.78 & - & - & 0.78 & - & 0.78 & - & 0.78 \\
\hline Desmodium scropiurus (Sw.) Desv. & - & - & - & - & 0.78 & - & - & - & - \\
\hline Digitaria horizontalis Willd & - & 0.78 & - & - & - & - & 0.78 & - & - \\
\hline Euphorbia heterophylla L. & - & 0.78 & - & - & - & - & - & - & - \\
\hline Gomphrena celosoides Mart. & 0.78 & 0.78 & 1.56 & 2.34 & 0.78 & 2.34 & 2.34 & 1.56 & 0.78 \\
\hline Ipomoea bartatas & - & - & - & - & - & - & - & - & 0.78 \\
\hline Ipomoea triloba Linn. & - & - & 0.78 & - & - & - & - & - & - \\
\hline Lapartea aestuans (Linn.) Chew. & 0.78 & - & 0.78 & - & 0.78 & - & - & - & 0.78 \\
\hline Leptochloa caerulescens Steud. & 0.78 & 0.78 & 0.78 & - & - & - & - & - & - \\
\hline Mariscus alternifolius Vahl & 0.78 & 1.56 & 0.78 & 0.78 & 0.78 & - & 1.56 & - & - \\
\hline Mitracarpus villosus (SW) DC & 0.78 & 2.34 & - & 1.56 & 1.56 & 0.78 & - & - & - \\
\hline Oldenladia corymbosa (Linn.)Roxb. & 0.78 & 1.56 & 0.78 & 2.34 & 0.78 & 1.56 & 0.78 & 0.78 & 0.78 \\
\hline Panicum maximum Jacq. O. Ktze & - & - & - & - & - & - & 0.78 & - & - \\
\hline Paspalum obiculare Forst. & - & - & - & - & - & - & - & - & 0.78 \\
\hline Paspalum conjugatum Berg. & 0.78 & - & - & - & - & - & - & - & - \\
\hline Peperomia pellucida (L.) H. B. \& K. & 0.78 & - & - & - & - & - & - & - & - \\
\hline Phillanthus amarus Schum, et Thonn. & - & - & - & - & - & 0.78 & - & - & - \\
\hline Platostoma africanum P. Beauv. & - & - & - & - & - & - & - & 0.78 & - \\
\hline Portulaca oleracea Linn. & - & - & - & - & - & - & - & 0.78 & - \\
\hline Physalis angulata Linn. & - & - & - & - & 0.78 & - & - & - & - \\
\hline Setaria barbata (Lam.) Kunth. & 0.78 & 0.78 & - & - & 1.56 & - & - & 0.78 & 0.78 \\
\hline Setaria longiseta P. Beauv. & - & - & - & 0.78 & - & - & 0.78 & - & - \\
\hline Synedrella nodiflora Gaertn. & - & - & 0.78 & 0.78 & 0.78 & 1.56 & 1.56 & - & - \\
\hline Talinum triangulare (Jacq.) Willd. & 0.78 & - & 1.56 & - & - & 0.78 & 1.56 & 0.78 & 1.56 \\
\hline Tridax procumbens $\mathrm{L}$. & 0.78 & 2.34 & 2.34 & 2.34 & 2.34 & 2.34 & 2.34 & 2.34 & 2.34 \\
\hline Waltheria indica Linn. & - & - & - & - & - & - & - & 0.78 & - \\
\hline Total number of weed species & 15 & 16 & 14 & 10 & 16 & 12 & 14 & 12 & 11 \\
\hline
\end{tabular}

Note: $\mathrm{MA}$ = Maize; CU = Cucumber; $\mathrm{SP}=$ Sweet Potato; $\mathrm{CO}=$ Corchorus; $\mathrm{LG}=$ Large Green; GA = Grain Amaranth; $\mathrm{MU}=$ Mucuna pruriens (var. utilis); $\mathrm{GN}=$ Groundnut; $\mathrm{C}=$ Control 


\section{Broadleaf weed density}

Broadleaf weed density was not significantly affected at 3 WAP (Tab. 4), however, at 6 WAP, significantly lower broadleaf weed densities were obtained in citrus/sweet potato, citrus/large green, control (sole citrus) and citrus/ cucumber plots $\left(28.67,45.00,50.00\right.$ and 76.33 weeds $\mathrm{m}^{-2}$ respectively). All these were significantly higher than what was obtained in citrus/groundnut plot $\left(143.00\right.$ weeds $\left.\mathrm{m}^{-2}\right)$. Citrus plots containing cucumber $\left(20.67\right.$ weeds $\left.\mathrm{m}^{-2}\right)$ and sweet potato $\left(21.00\right.$ weeds $\left.\mathrm{m}^{-2}\right)$ produced lower broadleaf weed densities at 9 WAP compared to control plot (26.33 weeds $\left.\mathrm{m}^{-2}\right)$, however, this effect was not significant.

\section{Grass weed density}

Grass weed density was generally low in all the citrus plots planted to these arable crops at all the periods of enumeration (Tab. 4). At 3 WAP, citrus/cucumber plot produced the lowest grass weed density $\left(3.00\right.$ weeds $\mathrm{m}^{-2}$ ) while citrus/maize plot produced the highest grass weed density $\left(15.67\right.$ weeds $\left.\mathrm{m}^{-2}\right)$ however, these means were not statistically significant. At 6 WAP, significantly lower grass weed densities were produced in citrus plots containing Mucuna and sweet potato $\left(0.33 \mathrm{~m}^{-2}\right.$ each $)$ than the control plot $\left(11.33 \mathrm{~m}^{-2}\right)$ and at 9 WAP, while citrus/cucumber plot produced grass weed density that was at par with control plot $\left(3.00 \mathrm{~m}^{-2}\right)$, citrus/grain amaranth plot had no grass weed recorded.

\section{Sedge weed density}

Sedge weed density was not significant in all the periods of enumeration however, at 3 WAP, citrus/grain amaranth plot produced the lowest sedge weed density (1.67 weeds $\mathrm{m}^{-2}$ ) while the highest was obtained in cucumber plot $\left(9.00\right.$ weeds $\left.\mathrm{m}^{-2}\right)$ (Tab. 4). At 6 WAP, citrus/sweet potato plot had no sedge weed at all while the control plot produced the highest $\left(18.00\right.$ weeds $\left.\mathrm{m}^{-2}\right)$ and at 9 WAP, citrus/Corchorus plot produced the lowest sedge weed density $\left(0.33\right.$ weeds $\left.\mathrm{m}^{-2}\right)$ and the highest value was obtained in the citrus plot containing grain/amaranth $(5.00$ weeds $\left.\mathrm{m}^{-2}\right)$.

Tab. 4. Effect of intercropping young citrus trees with arable crops on weed density $\left(\right.$ no $\left.\mathrm{m}^{-2}\right)$ at NIHORT, Nigeria

\begin{tabular}{|c|c|c|c|c|c|c|c|c|c|}
\hline \multicolumn{10}{|c|}{ Weed density $\left(\right.$ no $\left.^{-2}\right)$} \\
\hline \multirow{2}{*}{ Treatments } & \multicolumn{3}{|c|}{ Broadleaf weeds } & \multicolumn{3}{|c|}{ Grass weeds } & \multicolumn{3}{|c|}{ Sedges } \\
\hline & 3 WAP & $6 \mathrm{WAP}$ & 9 WAP & 3 WAP & 6 WAP & 9 WAP & 3 WAP & 6 WAP & 9 WAP \\
\hline Citrus/MA & 64.00 & $96.67 \mathrm{ab}$ & 87.00 & 15.67 & $3.33 b c$ & 1.67 & 5.00 & 4.00 & 4.33 \\
\hline Citrus/GN & 90.00 & $143.00 \mathrm{a}$ & 36.67 & 10.67 & $8.00 \mathrm{ab}$ & 0.33 & 4.52 & 13.33 & 1.33 \\
\hline Citrus/CU & 75.33 & $76.33 b$ & 20.67 & 3.00 & $1.33 \mathrm{bc}$ & 3.00 & 9.00 & 2.33 & 4.33 \\
\hline Citrus/LG & 98.33 & $45.00 \mathrm{~b}$ & 103.00 & 10.00 & $4.33 \mathrm{abc}$ & 2.00 & 5.67 & 0.67 & 1.67 \\
\hline Citrus/SP & 93.67 & $28.67 \mathrm{~b}$ & 21.00 & 8.00 & $0.33 c$ & 2.33 & 2.67 & 0.00 & 1.67 \\
\hline Citrus/GA & 135.67 & $168.68 \mathrm{ab}$ & 49.67 & 13.67 & $4.67 \mathrm{abc}$ & 0.00 & 1.67 & 17.67 & 5.00 \\
\hline Citrus/MU & 59.67 & $86.00 \mathrm{ab}$ & 46.33 & 11.33 & $0.33 c$ & 2.67 & 4.33 & 1.33 & 1.33 \\
\hline Citrus/CO & 103.33 & $104.33 \mathrm{ab}$ & 25.67 & 13.00 & $7.33 \mathrm{abc}$ & 0.67 & 3.33 & 10.33 & 0.33 \\
\hline Control & 90.00 & $50.00 \mathrm{~b}$ & 26.33 & 10.67 & $11.33 \mathrm{a}$ & 3.00 & 4.52 & 18.00 & 2.33 \\
\hline S.E.D. & 33.86 & 60.00 & 35.01 & 8.36 & 3.15 & 1.85 & 3.88 & 7.98 & 2.32 \\
\hline & $\mathrm{ns}$ & & $\mathrm{ns}$ & $\mathrm{ns}$ & & $\mathrm{ns}$ & $\mathrm{ns}$ & $\mathrm{ns}$ & $\mathrm{ns}$ \\
\hline
\end{tabular}

Note: MA = Maize; $\mathrm{SP}$ = Sweet Potato; GN = Groundnut; GA = Grain Amaranth; CU = Cucumber; Mucunapruriens (var. utilis); LG = Large Green Corchorusolitorius; WAP $=$ Weeks after planting

Tab. 5. Effect of intercropping young citrus trees with arable crops on weed dry weight $\left(\mathrm{g} \mathrm{m}^{-2}\right)$ in NIHORT, Ibadan, Nigeria

\begin{tabular}{|c|c|c|c|c|c|c|c|c|}
\hline \multicolumn{9}{|c|}{ Weed dry weight $\left(\mathrm{g} \mathrm{m}^{-2}\right)$} \\
\hline \multirow{2}{*}{ Treatments } & \multicolumn{3}{|c|}{ Broadleaf weeds } & \multicolumn{3}{|c|}{ Grass weeds } & \multicolumn{2}{|c|}{ Sedges } \\
\hline & 3 WAP & 6 WAP & 9 WAP & 3 WAP & 6 WAP & 9 WAP & 3 WAP & $6 \mathrm{WAP}$ \\
\hline Citrus/MA & $92.98 \mathrm{ab}$ & $44.44 \mathrm{bc}$ & 64.58 & $76.74 a$ & 3.57 & 4.17 & 10.71 & $6.22 b$ \\
\hline Citrus/GN & $83.08 \mathrm{ab}$ & $123.25 a$ & 58.80 & $17.40 \mathrm{ab}$ & 44.56 & 0.82 & 7.72 & $60.64 \mathrm{ab}$ \\
\hline Citrus/CU & $119.18 \mathrm{a}$ & $72.57 \mathrm{ab}$ & 77.39 & $7.62 b$ & 0.00 & 4.29 & 23.98 & $2.60 \mathrm{~b}$ \\
\hline Citrus/LG & $72.12 \mathrm{ab}$ & $16.51 \mathrm{c}$ & 66.86 & $4.84 \mathrm{~b}$ & 7.22 & 6.15 & 5.29 & $1.55 \mathrm{~b}$ \\
\hline Citrus/SP & $85.52 \mathrm{ab}$ & $34.82 \mathrm{bc}$ & 28.44 & $18.22 \mathrm{ab}$ & 0.00 & 0.85 & 7.51 & $0.00 \mathrm{~b}$ \\
\hline Citrus/GA & $45.72 b$ & $70.70 \mathrm{ab}$ & 48.47 & $1.68 \mathrm{~b}$ & 1.53 & 0.00 & 0.00 & $2.02 b$ \\
\hline Citrus/MU & $126.47 \mathrm{a}$ & $52.83 \mathrm{~b}$ & 37.06 & $3.14 b$ & 0.14 & 3.20 & 5.27 & $0.73 b$ \\
\hline Citrus/CO & $37.59 \mathrm{~b}$ & $53.36 b$ & 30.02 & $9.56 \mathrm{~b}$ & 3.59 & 1.07 & 1.63 & $4.86 \mathrm{~b}$ \\
\hline Control & $83.08 \mathrm{ab}$ & $62.26 \mathrm{~b}$ & 18.77 & $17.40 \mathrm{ab}$ & 27.53 & 0.00 & 7.77 & $64.63 a$ \\
\hline \multirow[t]{2}{*}{ S.E.D. } & 28.21 & 37.91 & 26.95 & 25.52 & 7.10 & 2.81 & 10.04 & 26.06 \\
\hline & & & ns & & $\mathrm{ns}$ & $\mathrm{ns}$ & ns & \\
\hline
\end{tabular}

Note: MA = Maize; SP = Sweet Potato; GN = Groundnut; GA = Grain Amaranth; CU = Cucumber Mucunapruriens (var. utilis); LG = Large Green Corchorusolitorius; WAP $=$ Weeks after planting 
136

\section{Broadleaf weed dry weight}

The various periods of enumeration of dry weed density were shown in Tab. 5. Citrus plot intercropped with Corchorus produced significantly lower dry weight (37.59 $\mathrm{g} \mathrm{m}^{-2}$ ) than the citrus plots containing Mucuna (126.47 $\mathrm{g} \mathrm{m}^{-2}$ ) at 3 WAP. Also significant effect was observed at 6 WAP where citrus/large green plots produced significantly lower broadleaf weed dry weight $\left(16.15 \mathrm{~g} \mathrm{~m}^{-2}\right)$ than citrus/groundnut plot $\left(123.25 \mathrm{~g} \mathrm{~m}^{-2}\right)$ while there were no significant effects at 9 WAP.

\section{Grass weed dry weight}

Grass weed dry weight was significantly lower in the citrus plots containing grain amaranth, Mucuna pruriens, large green, cucumber and Corchorus (1.68, 3.14, 4.84, 7.62 and $9.56 \mathrm{~g} \mathrm{~m}^{-2}$ respectively) than citrus/maize intercrop (76.74 $\mathrm{g} \mathrm{m}^{-2}$ ) at 3 WAP (Tab. 5). Citrus/cucumber and citrus/sweet potato plots at $6 \mathrm{WAP}$ and citrus/grain amaranth and control plots at 9 WAP recorded $0.0 \mathrm{~g} \mathrm{~m}^{-2}$ grass weed dry weight compared to citrus/groundnut plot at $6 \mathrm{WAP}$ and citrus/large green plots at 9 WAP (44.56 and $6.51 \mathrm{~g} \mathrm{~m}^{-2}$ respectively).

\section{Sedge weed dry weight}

Sedge dry weight was only significant at 6 WAP where sedge dry weights were less than $7 \mathrm{~g} \mathrm{~m}^{-2}$ in all the plots compared with control plot $\left(64.63 \mathrm{~g} \mathrm{~m}^{-2}\right)$ (Tab. 5) while there was no sedge in none of the plots at 9 WAP.

\section{Discussion}

The intercropping of young citrus trees with arable crops presented variable effects on weed parameters. The experimental site was infested with three different types of weeds which were broadleaf weeds, grass weeds and sedges. Broad leaf weeds comprising of 30 species accounted for $71.43 \%$ of the infestation followed by grass weed comprising of 10 species with $23.80 \%$ while sedges with only two species had the lowest $(4.76 \%)$. This high weed incidence may be attributed to the manual weed control method employed in the citrus orchard for about three years which is only effective before weeds produce flowers. In addition, the young citrus trees received moisture through drip irrigation in the dry season which some of these weeds might have taken advantage of for growth and development. The rate of occurrence of the weed species under the various treatments was less than $5 \%$, however, three weed species: G. celosoides, O. corymbosa and T. procumbens associated with all the intercrops except that of maize at 9 WAP and the consistent occurrence of $T$. procumbens with higher relative frequency $(2.34 \%)$ than the other two species is an indication of future dominance. This weed produces roots at the lower nodes and reproduces from seeds with an effective seed dispersal agent which will greatly affect its density in the future. Intercropping citrus with sweet potato, cucumber and large green significantly reduced broadleaf weed density compared to citrus/groundnut plot while the same trend was observed for grass weed density in the citrus plots containing sweet potato and M. pruriens var utilis than that obtained in the control plot. Similarly, both citrus/Mucuna and citrus/sweet potato plots produced significantly lower grass weed dry weight than the control plot at 6 WAP. This result agrees with the earlier reports of Jodha (1979) and George and Jeruto (2010).

\section{References}

Adewale JA, Oladosu LO, Laogun EA (1996). Factors limiting fruit tree production in South Western Nigeria: Implication for extension strategy. Proceeding of $14^{\text {th }}$ Annual Conference of Horticultural Society of Nigeria (HORTSON) $1^{\text {st }}-4^{\text {th }}$ April 1994, 200-204 p.

Aiyelaagbe IOO (2001). Productivity of an intercropped sweet orange orchard in Nigeria. Biol Agric Hortic 18:317-325

Bekunda M (1999). Farmers Responses to Soil Fertility Decline in Banana-based cropping Systems in Uganda. Managing Africa's Soils No. 4 Russel Publishers No Hingham, 2 p.

Bekunda M, Woomer PL (1996). Organic Resources Management in Banana-based cropping systems of the Lake Victoria Basin, Uganda. Agric, Eco Environ 59(3):171-180.

Clark E, Francis CA (1985). Transgressive yielding bean; maize intercrops interference in time and space Field Crops Res 11:37-53.

Florida Citrus Agriculture in the Classroom (2004). http: www. floridajuice.com/pdfs/teachers guide pdf.

George O, Jeruto P (2010). Sustainable horticultural crop production through intercropping: The case of fruits and vegetable crops: A review. Agric Biol J N Am 1(5):10981105.

Jodha NS (1979). Intercropping in traditional farming systems. Proc. Intl. Workshop on intercropping 10-13 Jan 1979 Hyderabad, India.

Natarajaa M, Nairk DM (1992). Competitive effects of short duration, bush type cowpea when intercropped with cotton in Zimbabwe. Expt Agric 28:41-48.

National Horticultural Research Institute (2000). 25 years of research into horticultural crops development in Nigeria (1975-2000), 140 p. In: Denton OA, Alasiri KO, Adejoro MA (Eds.). 25 ${ }^{\text {th }}$ Anniversary Commemoration Book.

Okigbo BN (1979). Evaluation for plant interactions and productivity in complex mixtures as a basis for improved cropping systems design, p. 350-356. In: Proc. Intl. Workshop on intercropping 10-13 Hyderbad, India.

Oyedele OO, Odeleye OMO, Olorunmaiye PM, Adebayo SO, Olaniyan AA, Alamu OO, Bamimore KM, Onyegbula U (2011). Preliminary studies on productivity response of spice and fruit vegetable crops in citrus juvenile orchard. Paper presented at the $29^{\text {th }}$ Annual Conference of Horticultural Society of Nigeria (HORTSON), 24-28 July, 2011.

Owuor C, Tenywa JS, Muwanga S, Woomer PL, Esele P (2002). Performance of a sorghum-legume intercrops in response to row levels of nitrogen Program and extended abstracts. Forum Working Agric Biol J N Am 1(5):1098-1105. 